\title{
Brain Regions Involved in Fractional Amplitude of Low-Frequency Fluctuation in Cluster Headache Patients: A Resting-State Functional MRI Study
}

\section{Yun Chen}

Chinese PLA General Hospital

\section{Xinbo Xing}

Fourth Medical Center of Chinese PLA General Hospital

Enchao Qiu

Chinese PLA General Hospital

Wei Dai

Chinese PLA General Hospital

\section{Lixia Tian}

Beijing Jiaotong University

\section{Zhao Dong}

Chinese PLA General Hospital

Shengyuan Yu ( $\nabla$ yusy1963@126.com )

Chinese PLA General Hospital

\section{Research Article}

Keywords: Cluster headache, resting-state functional magnetic resonance imaging, fractional amplitude of low-frequency fluctuation, brain activity

Posted Date: February 18th, 2022

DOI: https://doi.org/10.21203/rs.3.rs-1362271/v1

License: (c) (1) This work is licensed under a Creative Commons Attribution 4.0 International License. Read Full License 


\section{Abstract \\ Background:}

A limited number of studies have reported on fractional amplitude of low-frequency fluctuation (fALFF) in cluster headache $(\mathrm{CH})$. We used resting-state functional magnetic resonance imaging (RS-fMRI) to assess the possible pathogenic role of fALFF in $\mathrm{CH}$.

\section{Methods:}

RS-fMRI scans of 23 patients with $\mathrm{CH}$ were obtained (11with left-sided headache and 12 with right-sided headache), along with scans of 23 age- and sex-matched normal controls. The RS-fMRI data were analyzed to explore abnormal brain activity in the left $\mathrm{CH}$ and right $\mathrm{CH}$ patients during the non-painful state of cluster period. fALFF was compared between patients and controls, and correlation analysis between the regional mean fALFF values and clinical characteristics was performed.

\section{Results:}

A decrease in fALFF was detected in the left cerebellum, left lentiform nucleus, left frontal lobe, left anterior cingulate, and right postcentral gyrus in the left $\mathrm{CH}$ group compared to the controls, while a decrease of fALFF was detected in the right cerebellum, right cingulate gyrus, right superior parietal lobule, right inferior parietal lobule, right postcentral gyrus, and left precuneus in the right $\mathrm{CH}$ group. No patient had a region with increased fALFF. A moderate correlation was observed between some regional mean fALFF values and the clinical characteristics.

\section{Conclusions:}

We deduced that dysfunction in multiple brain areas is involved in the non-painful state of $\mathrm{CH}$ during cluster period. Several functional networks are involved, such as the default-mode, cerebellar, and somatomotor networks, suggesting that $\mathrm{CH}$ is the result of abnormal functioning in multiple brain functional networks.

\section{Background}

Cluster headache $(\mathrm{CH})$ is a male-predominant [1], excruciating and strictly one-sided pain syndrome characterized by attacks accompanied by marked ipsilateral cranial autonomic symptoms, such as lacrimation and conjunctival injection [2]. $\mathrm{CH}$ affects about $0.12 \%$ of the population [3]. Single attacks last 15-180 min and the frequency of attacks ranges from once every day to eight times per day. $\mathrm{CH}$ is considered a chronobiological disorder, where seasonal and circadian rhythmicity affect the likelihood of an attack [4]. Approximately $80-90 \%$ of $\mathrm{CH}$ patients suffer from episodic headaches [5]. These clinical 
features indicate that $\mathrm{CH}$ is a central disease, activated by the posterior hypothalamus [6] and regulated by the trigemino-parasympathetic reflex [7]. Several intracranial cortical brain regions are also involved, but the pathogenesis of $\mathrm{CH}$ remains unclear [8]. Functional neuroimaging of headache patients has revolutionized our understanding of the pathophysiology of $\mathrm{CH}$ and provided unique insight into this syndrome [9].

As a resting-state functional magnetic resonance imaging (RS-fMRI) signal, fractional amplitude of lowfrequency fluctuation (fALFF), which covers the low-frequency power spectrum $(0.01-0.08 \mathrm{~Hz})$, reflects the intensity of local spontaneous activity in brain areas [10, 11]. fALFF is minimally affected by the cerebrospinal fluid, veins, and physiological noise, and improves the cortical activity detection rate. fALFF is sensitive and specific to spontaneous neuronal activity of the resting brain [12]. fALFF is a powerful marker of group differences in spontaneous brain activity, and brain areas with increased fALFF are associated with the default mode network (DMN) in the resting state $[13,14]$. fALFF is now considered reliable for measuring regional coherence in spontaneous activity, and for revealing the pathophysiology of neuropsychiatric disorders including Alzheimer's disease [15], post-stroke depression [16], anxiety, depression [17], and migraine [18, 19].

However, few studies have detected spontaneous brain activity in patients with $\mathrm{CH}$ using fALFF during the resting state. We performed RS-fMRI during the non-painful period of $\mathrm{CH}$, calculated the fALFF value, and compared brain activity between $\mathrm{CH}$ patients and normal controls.

\section{Methods}

\section{Participants}

All procedures were approved by the Chinese Ministry of Health and the Ethics Committee of the Chinese PLA General hospital, Beijing, china and were conducted according to the ethical principles of the Declaration of Helsinki. Participants admitted to the International Headache Center of PLA General Hospital between January 2017 and January 2018 were enrolled in the study after providing written informed consent.

The inclusion criteria were as follows: met the diagnostic criteria for $\mathrm{CH}$ of the International Classification of Headache Disorders 3rd Edition (ICHD-3) [20]; severe unilateral headache; aged 20-60 years; righthanded; no chronic diseases, including diabetes, hypertension, hypercholesterolemia, cardiovascular and cerebrovascular diseases, or tumors, epilepsy, infectious diseases, connective tissue diseases, other types of chronic pain, or severe anxiety and depression; and no history of alcohol, nicotine, or other substance abuse. The control group was healthy people recruited from the staff of the PLA General Hospital and their relatives. The normal controls (NC) were age- and gender-matched with the $\mathrm{CH}$ patients and had no history of primary headache or any other type of headache in the past year. This study was reviewed and approved by the Chinese Ministry of Health and the Ethics Committee of the Chinese PLA General hospital, Beijing, china and all participants signed an informed consent form. 


\section{RS-fMRI data collection}

All subjects were advised to keep their eyes closed, but to remain awake and think of nothing in particular during the scan. The scans were taken with the Sigma 3.0 T magnetic resonance imaging system (Siemens, Munich, Germany), and the functional images were acquired using an echo plane pulse sequence. The scanning parameters were as follows: repetition time $(T R)=2,000 \mathrm{~ms}$, echo time $(T E)=30$ ms, layer thickness $=3.0 \mathrm{~mm}$, interval $=0.6 \mathrm{~mm}$, matrix $=64 \times 64$, field of view $=200 \times 200 \mathrm{~mm}$, flip angle $=90^{\circ}$, scan duration $=4 \mathrm{~min} 14 \mathrm{~s}$. There were 33 layers, from the foramen magnum to the top of the head (240 time points and 7,920 image frames). Subsequently, a sagittal 3D Tl-weighted image of the whole brain was acquired in 128 layers $(T R=2,500 \mathrm{~ms}, T E=3.5 \mathrm{~ms}$, layer thickness $=1.0 \mathrm{~mm}$, interval $=0.6 \mathrm{~mm}$, matrix $=256 \times 256$, field of view $=256 \times 256 \mathrm{~mm}$, flip angle $\left.=8^{\circ}\right)$.

\section{Image data preprocessing}

MATLAB 2013b software (MathWorks, Natick, MA, USA) was used for data processing, and the fMRI data were processed using DPARSF software (http://www.rfmri.org). The fMRI data preprocessing method employed herein is well-established [17] and includes eight steps. First, DICOM format data were converted into the NIFTI format (Neuroimaging Informatics Technology Initiative). Second, the first 10 time points were removed to reduce the effect of maladjustments at the beginning of the scan. Then, data from all layers were calibrated to a specific time point using a time correction process, and the functional time series was realigned to correct for head motion across the time series. The brain images of each subject were then transferred to standard space for normalization, to reduce differences between individuals for the next step, i.e., smoothing, in which the effect of spatial noise and differences in brain structure between subjects was reduced using a $4 \times 4 \times 4 \mathrm{~mm}$ smoothing kernel. Finally, after detrending, the low-frequency band wave was used to remove the effect of high frequency signals from respiratory heartbeats, and high frequency noise, to obtain the low-frequency $(0.01-0.10 \mathrm{~Hz})$ fluctuations in the resting brain (filtering). After calculating the fALFF value, the Gaussian kernel function was applied for spatial smoothing, and a standardized fALFF diagram was acquired of each participant over the range of $0.01-0.08 \mathrm{~Hz}$. Then, Statistical Parametric Mapping 8 (SPM8) (http://www.fil.ion.ucl.ac.uk/spm) and RESTplus V1.21 (http://www.restfmri.net/forum/REST) software were used for statistical analyses of the fALFF data, and to integrate the fALFF value with the image and determine changes in local brain function. The abnormal brain area of $\mathrm{CH}$ patients was defined as the mask, and RESTplus was used to extract the corresponding index values from the center of the region of interest (radius $=6 \mathrm{~mm}$ ), to derive the mean fALFF value.

\section{Statistical analysis}

Headache pain was evaluated using a visual analog scale (VAS). The chi-square test or Fisher's exact test, performed with SPSS 18.0 software (SPSS Inc., Chicago, IL, USA), was used to analyze the demographic and clinical characteristics of the patients. Independent sample tests were performed using Matlab (R2013b) to compare fALFF values between the case and control groups. RESTplus software was used to extract the corresponding index values. A $P$-value $<0.01$ for a minimum volume of $1,458 \mathrm{~mm}^{3}$ 
was taken to indicate a significant difference. Pearson's correlation analysis was performed to assess the relationship between the mean fALFF value across all voxels (in brain regions with abnormal fALFF values) and clinical characteristics of the $\mathrm{CH}$ patients.

\section{Results}

\section{Demographic characteristics}

Forty-six volunteers were enrolled in the study, including $23 \mathrm{CH}$ patients and 23 healthy sex- and agematched controls. The healthy controls did not have any primary or secondary head or face pain. More male than female $\mathrm{CH}$ patients suffered from $\mathrm{CH}$, in both the left and right $\mathrm{CH}$ groups $(81.8 \%$ and $75.0 \%$ male, respectively). The average age and age at onset of the patients in the left and right $\mathrm{CH}$ groups were 33.8 and 33.2 years, and 26.7 and 23.5 years, respectively. The majority of the patients experienced severe pain (VAS score $>8$ ), lasting for more than $30 \mathrm{~min}$ and accompanied by conjunctive ingestion and lacrimation at the time of onset. No significant differences in gender, age, age at onset, duration of headache attack, number of attacks per day, cluster bout duration, pain duration, degree of pain at onset, or accompanying symptoms including nausea, vomiting, photophobia, phonophobia, conjunctive ingestion, lacrimation, nasal congestion, and rhinorrhea were observed between the left and right $\mathrm{CH}$ groups (Table 1). 
Table 1

Comparison of the general characteristics of the left and right cluster headache groups

\begin{tabular}{|c|c|c|c|c|}
\hline Characteristic & & $\begin{array}{l}\text { Left } \\
(n=11)\end{array}$ & $\begin{array}{l}\text { Right } \\
(n=12)\end{array}$ & $p$ \\
\hline \multirow[t]{2}{*}{ Age (years) } & Mean \pm SD & $33.8 \pm 12.8$ & $33.2 \pm 9.1$ & 0.275 \\
\hline & Onset age & $26.7 \pm 10.1$ & $23.5 \pm 7.1$ & 0.085 \\
\hline \multirow[t]{2}{*}{ Sex } & Female & 2 & 3 & 1 \\
\hline & Male & 9 & 9 & \\
\hline \multirow[t]{2}{*}{ Headache attack duration } & $\leq 30 \min$ & 1 & 2 & 1 \\
\hline & $>30 \mathrm{~min}$ & 10 & 10 & \\
\hline \multirow[t]{2}{*}{ Frequency of attacks } & $\leq 1$ time/day & 8 & 10 & 0.640 \\
\hline & $>1$ time/day & 3 & 2 & \\
\hline \multirow[t]{2}{*}{ Cluster bout duration } & $\leq 4$ weeks & 5 & 7 & 0.537 \\
\hline & $>4$ weeks & 6 & 5 & \\
\hline \multirow[t]{2}{*}{ Disease duration } & $\leq 10$ years & 7 & 5 & 0.292 \\
\hline & $>10$ years & 4 & 7 & \\
\hline \multirow[t]{2}{*}{ Visual analogue scale score } & $\leq 8$ & 5 & 2 & 0.193 \\
\hline & $>8$ & 6 & 10 & \\
\hline \multirow[t]{2}{*}{ Nausea } & Yes & 8 & 6 & 0.400 \\
\hline & No & 3 & 6 & \\
\hline \multirow[t]{2}{*}{ Vomiting } & Yes & 6 & 3 & 0.214 \\
\hline & No & 5 & 9 & \\
\hline \multirow[t]{2}{*}{ Photophobia } & Yes & 5 & 3 & 0.400 \\
\hline & No & 6 & 9 & \\
\hline \multirow[t]{2}{*}{ Phonophobia } & Yes & 4 & 3 & 0.667 \\
\hline & No & 7 & 9 & \\
\hline \multirow[t]{2}{*}{ Conjunctive ingestion } & Yes & 10 & 11 & 1 \\
\hline & No & 1 & 1 & \\
\hline Lacrimation & Yes & 9 & 12 & 0.217 \\
\hline
\end{tabular}




\begin{tabular}{|lllll|}
\hline Characteristic & $\begin{array}{l}\text { Left } \\
(\mathbf{n = 1 1 )}\end{array}$ & $\begin{array}{l}\text { Right } \\
(\mathbf{n}=12)\end{array}$ & $\boldsymbol{p}$ \\
\hline No & 2 & 0 & \\
\hline Nasal congestion & Yes & 4 & 8 & 0.146 \\
\hline Rhinorrhea & No & 7 & 4 & \\
\hline & Yes & 5 & 9 & 0.214 \\
\hline
\end{tabular}

\section{fALFF results}

fALFF values of the left cerebellum, left lentiform nucleus, left frontal lobe, left anterior cingulate, and right postcentral gyrus were significantly lower in the left headache than NC group, while fALFF values of the right cerebellum, right cingulate gyrus, right superior parietal lobule, right inferior parietal lobule, right postcentral gyrus, and left precuneus were significantly lower in the right headache than NC group $(P<$ 0.01). No fALFF value in the left $\mathrm{CH}$ or right $\mathrm{CH}$ groups was higher than the corresponding value in the $\mathrm{NC}$ group (Tables 2 and 3).

Table 2

The fALFF brain area was decreased in the left $\mathrm{CH}$ compared to the normal control

\begin{tabular}{|c|c|c|c|c|c|c|c|}
\hline \multirow[t]{2}{*}{ Brain regions } & \multirow[t]{2}{*}{ Hemisphere } & \multirow[t]{2}{*}{ BA } & \multirow[t]{2}{*}{$\begin{array}{l}\text { No. of } \\
\text { voxels }\end{array}$} & \multicolumn{3}{|c|}{$\begin{array}{l}\text { Talairach coordinates } \\
(\mathrm{mm})\end{array}$} & \multirow[t]{2}{*}{$\begin{array}{l}\text { T-score } \\
\text { (peak) }\end{array}$} \\
\hline & & & & $\mathbf{x}$ & $y$ & $\mathbf{z}$ & \\
\hline Left cerebellum & L & - & 17 & -27 & -51 & -33 & -3.473 \\
\hline $\begin{array}{l}\text { Lentiform } \\
\text { nucleus }\end{array}$ & L & - & 47 & -24 & 3 & -6 & -4.4634 \\
\hline Frontal lobe & L & 10 & 29 & -24 & 33 & 6 & -3.6416 \\
\hline Anterior cingulate & L & 24 & 44 & -18 & 36 & 18 & -4.3421 \\
\hline $\begin{array}{l}\text { Postcentral_R } \\
\text { (aal) }\end{array}$ & $\mathrm{R}$ & 3 & 16 & 27 & -33 & 51 & -4.5212 \\
\hline
\end{tabular}


Table 3

The fALFF brain area was decreased in the right $\mathrm{CH}$ compared to the normal control

\begin{tabular}{|c|c|c|c|c|c|c|c|}
\hline \multirow[t]{2}{*}{ Brain regions } & \multirow[t]{2}{*}{ Hemisphere } & \multirow[t]{2}{*}{ BA } & \multirow[t]{2}{*}{$\begin{array}{l}\text { NO. of } \\
\text { voxels }\end{array}$} & \multicolumn{3}{|c|}{$\begin{array}{l}\text { Talairach coordinates } \\
(\mathrm{mm})\end{array}$} & \multirow[t]{2}{*}{$\begin{array}{l}\text { T-score } \\
\text { (peak) }\end{array}$} \\
\hline & & & & $\mathbf{x}$ & $y$ & $\mathbf{z}$ & \\
\hline Right cerebellum & $\mathrm{R}$ & - & 68 & 45 & -66 & -42 & -4.7791 \\
\hline Cingulate gyrus & $\mathrm{R}$ & 31 & 109 & 3 & -30 & 39 & -5.6906 \\
\hline $\begin{array}{l}\text { Superior parietal } \\
\text { lobule }\end{array}$ & $\mathrm{R}$ & 7 & 66 & 36 & -60 & 51 & -4.7168 \\
\hline $\begin{array}{l}\text { Inferior parietal } \\
\text { lobule }\end{array}$ & $\mathrm{R}$ & 40 & 81 & 48 & -45 & 48 & -4.697 \\
\hline Precuneus_L (aal) & L & 7 & 96 & -9 & -60 & 54 & -4.3352 \\
\hline Postcentral gyrus & $\mathrm{R}$ & 3 & 54 & 15 & -60 & 72 & -4.4526 \\
\hline
\end{tabular}

\section{Correlation analysis of clinical characteristics and mean fALFF values}

No significant correlations were observed between the regional fALFF values and the clinical characteristics. However, some regional fALFF values and clinical characteristics were moderately correlated. For example, a moderate negative correlation was observed between the left lenticular nucleus fALFF values and VAS score in the left $\mathrm{CH}$ group $(r=-0.691, P=0.019)$. The right superior parietal lobule fALFF values were moderately correlated with the number of attacks per day in the right $\mathrm{CH}$ group ( $r=-$ $0.665, P=0.018)$, and the right inferior parietal lobule fALFF values were moderately correlated with patient age $(r=0.620, P=0.032)$. Additionally, a moderate correlation was detected between the right postcentral gyrus fALFF values and typical duration of the bout (in weeks) $(r=0.602, P=0.038)$ (Fig. 3).

\section{Discussion}

To the best of our knowledge, this is the first RS-fMRI investigation of patients with $\mathrm{CH}$ to use fALFF values. We found a decrease in fALFF values in certain brain areas, such as the cerebellum, lentiform nucleus, frontal lobe, and right postcentral gyrus, indicating that the intensity of local functional activity was lowered in several brain networks, such as the DMN, cerebellar network (CEN), and somatomotor network (SEN). Our results suggest that the function of multiple brain networks in the non-painful $\mathrm{CH}$ period is impaired.

Because $\mathrm{CH}$ attacks are usually unilateral, we divided the patients into left and right headache groups. Patients in the left headache group showed only left headache during the attack, while only right pain 
was observed in the right group. The abnormally active brain areas were not the same between the left and right headache groups. This may be due to differences in structure [21] and function [22] between the bilateral cerebral hemispheres. However, fMRI research has demonstrated that cerebral asymmetries are never absolute; even for strongly left-lateralized functions such as language, the right hemisphere makes a significant contribution [23]. Second, brain network functions in bilateral hemispheres are not completely symmetrical. Imaging, clinical, and behavioral data demonstrate hemispheric asymmetries in attentional networks, as revealed by the lateralized attention network test [24]. The orienting and alerting networks show left hemisphere dominance [25], while the executive control network is right hemispheredominant [26]. The allocation of spatial attention is balanced through the regulatory action of the corpus callosum. An impairment in the lateralization of brain function is seen in many diseases; for example, the visuospatial attention network may be disrupted after stroke and rehabilitated by non-invasive brain stimulation [27]. Therefore, we believe that studies of $\mathrm{CH}$ should group participants according to headache side.

Based on regional homogeneity, we previously showed that activity in brain regions such as the bilateral middle prefrontal cortex, right posterior cingulate cortex, and left dorsolateral prefrontal cortex decreases during a cluster period [28]. Another study reported greater hypometabolism in the perigenual anterior cingulate, prefrontal and orbitofrontal cortices, as revealed by positron emission tomography, in $\mathrm{CH}$ patients compared to healthy volunteers [29], which supported our results. Notably, the cingulate gyrus, superior parietal lobule, inferior parietal lobule, precuneus, and frontal lobe constitute the hub region of the DMN, which is the most studied brain network, was shown to be active in the resting state (i.e., in the absence of thinking), but was dormant in the task state [30]. In our study, activity of the default network in $\mathrm{CH}$ patients decreased rather than increased, suggesting that the activity of the $\mathrm{DMN}$ in $\mathrm{CH}$ patients is reduced on the same side as the headache during the resting state.

This study showed that both left- and right-sided $\mathrm{CH}$ were associated with decreased activity in the ipsilateral cerebellum. Although the specific mechanism underlying the involvement of the cerebellum in $\mathrm{CH}$ remains unclear, a previous structural and functional imaging study suggested that the cerebellum might be involved in the pathogenesis of $\mathrm{CH}$ [31]. Naegel et al. reported that the volume of cerebellar gray matter was greater in $\mathrm{CH}$ patients compared to $\mathrm{NC}$ [32]. This increase in gray matter may reflect increased regional cortical plasticity and promote chronic $\mathrm{CH}$, as indicated by a structural imaging study of patients with paroxysmal and chronic $\mathrm{CH}$ [33]. A study of the macroscale functional neuroanatomy of the cerebellum revealed causal interactions of sub-lobular structures in the CEN with a large-scale cortical network and the DMN in a non-motor learning paradigm [34]. Morelli et al. were the first to study brain activation patterns in patients with episodic $\mathrm{CH}$ using RS-fMRI, and reported that the cerebellum, as an "unconventional" pain-related brain area, was abnormally activated [31]. Farago et al. used fMRI to study brain activation patterns and intensity in $\mathrm{CH}$ patients during the non-painful period. They also found that the ipsilateral cerebellum and CEN of headache patients were functionally connected, i.e., were activated simultaneously [35]. However, in this study, a decrease in cerebellar activation on the side ipsilateral to the pain was seen. There are several possible explanations for the abnormalities observed in our patients. First, we speculate that this may have been due to the research methods used. In the former study, 
independent component analysis of functional integration was performed, while we used fALFF for functional differentiation. In addition, the acquisition range of the low-frequency blood oxygen signal was different: a "low-frequency" blood sample was collected $(0.02-0.01 \mathrm{~Hz})$ in the former study, while in this study the frequency range was $0.01-0.08 \mathrm{~Hz}$. Furthermore, cerebellar activity occurs during the presence of acute and chronic pain [36], and the occurrence of headache in patients with $\mathrm{CH}$ is a dynamic process. We speculate that cerebellar activity is also dynamically changed. Therefore, we hypothesized that the CEN is involved in the painful period of $\mathrm{CH}$.

The fALFF values in the right postcentral gyrus decreased in both of our groups. A moderate correlation was detected between the right postcentral gyrus fALFF value and headache attack duration in the right $\mathrm{CH}$ group. The central posterior gyrus is in the primary somatosensory area and somatosensory network, which is mainly involved in the localization and recognition of pain, and receives signals from the thalamus [37]. Both the current study and a previous one observed that postcentral gyrus activity decreased in chronic pain, which may be related to the suppression of pain perception under long-term stimulation [38].

The activity of the left lenticular nucleus decreased in our left $\mathrm{CH}$ headache group. In addition, a negative correlation was observed between the fALFF value in the left lenticular nucleus and VAS score; the lower the fALFF value, the higher the VAS score, and the more severe the pain. A study that utilized voxel-based morphometry to evaluate abnormal patterns of local gray and white matter in patients with $\mathrm{CH}$ reported that the volume of the lenticular nucleus was decreased in patients with $\mathrm{CH}$ [39]. Previous studies on the subcortical microstructure of right and left $\mathrm{CH}$ patients reported "higher" diffusion parameters of the lenticular nucleus [40], indicating microstructural disintegration and atrophy. The basal ganglia and subcortical structures have been proposed to play a central role in nociception. Furthermore, basal ganglia structures are activated during the application of painful stimuli [41]. Also, reduced fractional anisotropy was found in the corpus callosum and some frontal and parietal white matter tracts in $\mathrm{CH}$ patients, mainly on the contralateral side of the pain [42]. We speculate that the fALFF value of the lenticular nucleus may be useful when choosing the $\mathrm{CH}$ treatment during the non-painful $\mathrm{CH}$ period.

The activity of the parietal lobe was decreased in our right $\mathrm{CH}$ group, including the right superior parietal lobule, right inferior parietal lobule, right postcentral gyrus, and left precuneus lobe. A brain network study reported that the functional connections between the parietal lobe, insular lobe, and cerebellum were significantly stronger in $\mathrm{CH}$ than migraine patients, in both RS-fMRI and task-state fMRI analyses [43]. A study based on voxel-based morphometry reported that the grey matter volume of the left inferior parietal lobule was decreased, while the grey matter volume of the right cuneus was increased, in patients with $\mathrm{CH}$ [39]. The right superior parietal lobule fALFF value was moderately correlated with the frequency of attacks. The greater the frequency of attacks, the lower the right superior parietal lobule fALFF value. We speculate that the frequency of attacks in $\mathrm{CH}$ patients may not only affect the function of the superior parietal lobule, but also the structure of the brain. 
Our findings have several potential implications for clinical treatment. Currently used analgesics, as well as oxygen, are not completely effective for $\mathrm{CH}$. Given the hypofunctioning of the brain network on the side ipsilateral to the during period of $\mathrm{CH}$, could non-invasive treatments be used? For example, meditation changes the activity of the DMN and improves the coupling of multiple brain networks by changing the functional connection between brain regions [44]. Transcranial magnetic stimulation (TMS) increases brain activity through single or repetitive pulses, which affect neuroplasticity and the functional connections among brain networks [45]. A naturalistic study reported that treating chronic $\mathrm{CH}$ with maintenance sessions of repetitive TMS (rTMS) of the motor cortex reduced the intensity of permanent and paroxysmal pain, as well as the daily number of painful attacks. However, there are few reports on the use of rTMS to treat $\mathrm{CH}$ [46]. Our results may lead to less-invasive treatments for $\mathrm{CH}$.

Our study had several limitations. First, the sample size was small. The rarity of $\mathrm{CH}$ makes it difficult to conduct large, well-controlled studies. Second, our study was only concerned with the non-painful period of $\mathrm{CH}$, as dynamic changes in fALFF during the onset of $\mathrm{CH}$ cannot be observed. Thus, a longitudinal follow-up study may be necessary; more evidence is needed to confirm that the changes in spontaneous brain activity observed herein were the result of $\mathrm{CH}$.

\section{Conclusion}

Our results provide new insight into the pathogenesis of $\mathrm{CH}$. Although the pathogenesis of $\mathrm{CH}$ is highly complex, dysfunction in multiple brain areas, such as the DMN, CEN, and SEN, was involved in the nonpainful $\mathrm{CH}$ period. It is necessary to study $\mathrm{CH}$ according to the headache side, given the lateralization of brain function.

\section{List Of Abbreviations}

$\mathrm{CH}$

cluster headache

fALFF

fractional amplitude of low-frequency fluctuation RS-FMRI resting-state functional magnetic resonance imaging NC

The normal Controls

DMN default-mode network

CEN

cerebellar network

SEN

somatomotor network VAS 
visual analog scale

TMS

Transcranial magnetic stimulation

BA

Brodmann's area.

\section{Declarations}

\section{Ethics approval and consent to participate}

The Institutional Review Board of the Chinese PLA General Hospital approved the research protocol, and the procedures conformed to the tenets of the Declaration of Helsinki.

\section{Consent for publication}

Not applicable.

\section{Availability of data and materials}

The datasets used and/or analysed during the current study are available from the corresponding author on reasonable request.

\section{Competing interests}

The authors declare that they have no competing interests

\section{Funding}

This work was supported by the National Natural Science Foundation of China (grants 82071226ه $82171208,81771200 \rrbracket 81901145 \llbracket 81901134 \rrbracket$

\section{Authors' contributions}

$Y C, X X, Z D, E Q$ and SY contributed the conception and design of the study. EQ, WD, ZD, YC, XX and LT contributed to MR data acquisition, and $X X, Y C$ contributed the clinical data acquisition. $Y C$, EQ and $L T$ contributed the image analysis and statistical analysis, $Y C, Z D$ and $X X$ contributed to the interpretation and draft. YC, ZD, EQ, LT and SY contributed to the revision for important intellectual content. All authors read and approved the final manuscript.

\section{Acknowledgements}

The authors thank all the participants and study sites that took part in this study.

\section{References}


1. Ekbom K, Svensson DA, Traff H et al (2002) Age at onset and sex ratio in cluster headache: observations over three decades. Cephalalgia 22:94-100. DOl:https://doi.org/10.1046/j.14682982.2002.00318.x

2. Hoffmann J, May A (2018) Diagnosis, pathophysiology, and management of cluster headache. Lancet Neurol 17:75-83. DOl:https://doi.org/10.1016/S1474-4422(17)30405-2

3. Fischera M, Marziniak M, Gralow I et al (2008) The incidence and prevalence of cluster headache: a meta-analysis of population-based studies. Cephalalgia 28:614-618.

DOl:https://doi.org/10.1111/j.1468-2982.2008.01592.x

4. Lee MJ, Cho SJ, Park JW et al (2020) Temporal changes of circadian rhythmicity in cluster headache. Cephalalgia 40:278-287. DOI:https://doi.org/10.1177/0333102419883372

5. Torelli P, Beghi E, Manzoni GC (2005) Cluster headache prevalence in the Italian general population. Neurology 2005, 64:469-474. DOl:https://doi.org/10.1212/01.WNL.0000150901.47293.BC

6. May A, Goadsby PJ (2001) Hypothalamic involvement and activation in cluster headache. Curr Pain Headache Rep 5:60-66. DOI:https://doi.org/10.1007/s11916-001-0011-4

7. Leone M, Proietti Cecchini A (2017) Advances in the understanding of cluster headache. Expert Rev Neurother 17:165-172. DOI:https://doi.org/10.1080/14737175.2016.1216796

8. Ha SY, Park KM (2019) Alterations of structural connectivity in episodic cluster headache: A graph theoretical analysis. J Clin Neurosci 62:60-65. DOI:https://doi.org/10.1016/j.jocn.2019.01.007

9. Iacovelli E, Coppola G, Tinelli E et al (2012) Neuroimaging in cluster headache and other trigeminal autonomic cephalalgias. J Headache Pain 13:11-20. DOI:https://doi.org/10.1007/s10194-011-04038

10. Mantini D, Perrucci MG, Del Gratta C et al (2007) Electrophysiological signatures of resting state networks in the human brain. Proc Natl Acad Sci USA 104:13170-13175.

DOl:https://doi.org/10.1073/pnas.0700668104

11. Zang YF, He Y, Zhu CZ et al (2007) Altered baseline brain activity in children with ADHD revealed by resting-state functional MRI. Brain Dev 29:83-91.

DOl:https://doi.org/10.1016/j.braindev.2006.07.002

12. Chen YC, Xia W, Luo B et al (2015) Frequency-specific alternations in the amplitude of low-frequency fluctuations in chronic tinnitus. Front Neural Circuits 9:67.

DOl:https://doi.org/10.3389/fncir.2015.00067

13. Zou QH, Zhu CZ, Yang Y et al (2008) An improved approach to detection of amplitude of lowfrequency fluctuation (ALFF) for resting-state fMRI: fractional ALFF. J Neurosci Methods 172:137141. DOl:https://doi.org/10.1016/j.jneumeth.2008.04.012

14. Zuo XN, Di Martino A, Kelly C et al (2010) The oscillating brain: complex and reliable. Neuroimage 49:1432-1445. DOl:https://doi.org/10.1016/j.neuroimage.2009.09.037

15. Yang L, Yan Y, Wang Y et al (2018) Gradual Disturbances of the Amplitude of Low-Frequency Fluctuations (ALFF) and Fractional ALFF in Alzheimer Spectrum. Front Neurosci 12:975. DOl:https://doi.org/10.3389/fnins.2018.00975 
16. Egorova N, Veldsman M, Cumming T et al (2017) Fractional amplitude of low-frequency fluctuations (fALFF) in post-stroke depression. Neuroimage Clin 16:116-124.

DOI:https://doi.org/10.1016/j.nicl.2017.07.014

17. Shu Y, Kuang L, Huang Q et al (2020) Fractional amplitude of low-frequency fluctuation (fALFF) alterations in young depressed patients with suicide attempts after cognitive behavioral therapy and antidepressant medication cotherapy: A resting-state fMRI study. J Affect Disord 276:822-828. DOI:https://doi.org/10.1016/j.jad.2020.07.038

18. Kim YE, Kim MK, Suh SI et al (2021) Altered trigeminothalamic spontaneous low-frequency oscillations in migraine without aura: a resting-state fMRI study. BMC Neurol 21:342. DOl:https://doi.org/10.1186/s12883-021-02374-7

19. Wang JJ, Chen X, Sah SK et al (2016) Amplitude of low-frequency fluctuation (ALFF) and fractional ALFF in migraine patients: a resting-state functional MRI study. Clin Radiol 71:558-564. DOl:https://doi.org/10.1016/j.crad.2016.03.004

20. Headache Classification Committee of the International Headache Society (IHS) (2018) The International Classification of Headache Disorders, 3rd edition. Cephalalgia 38:1-211. DOl:https://doi.org/10.1177/0333102417738202

21. Foundas AL, Faulhaber JR, Kulynych JJ et al (1999) Hemispheric and sex-linked differences in Sylvian fissure morphology: a quantitative approach using volumetric magnetic resonance imaging. Neuropsychiatry Neuropsychol Behav Neurol 12:1-10.

22. Corballis MC (2021) Humanity and the left hemisphere: The story of half a brain. Laterality 26:1933. DOI:https://doi.org/10.1080/1357650X.2020.1782929

23. Tailby C, Abbott DF, Jackson GD (2017) The diminishing dominance of the dominant hemisphere: Language fMRI in focal epilepsy. Neuroimage Clin 14:141-150. DOI:https://doi.org/10.1016/j.nicl.2017.01.011

24. Greene DJ, Barnea A, Herzberg K et al (2008) Measuring attention in the hemispheres: the lateralized attention network test (LANT). Brain Cogn 66:21-31.

DOI:https://doi.org/10.1016/j.bandc.2007.05.003

25. Asanowicz D, Marzecova A, Jaskowski P et al (2012) Hemispheric asymmetry in the efficiency of attentional networks. Brain Cogn 79:117-128. DOl:https://doi.org/10.1016/j.bandc.2012.02.014

26. Hampshire A, Chamberlain SR, Monti MM et al (2010) The role of the right inferior frontal gyrus: inhibition and attentional control. Neuroimage 50:1313-1319.

DOl:https://doi.org/10.1016/j.neuroimage.2009.12.109

27. Zebhauser PT, Vernet M, Unterburger E et al (2019) Visuospatial Neglect - a Theory-In formed Overview of Current and Emerging Strategies and a Systematic Review on the Therapeutic Use of Non-invasive Brain Stimulation. Neuropsychol Review 29:397-420.

DOl:https://doi.org/10.1007/s11065-019-09417-4

28. Qiu EC, Yu SY, Liu RZ et al (2012) Altered regional homogeneity in spontaneous cluster headache attacks: a resting-state functional magnetic resonance imaging study. Chin Med J 125:705-709. 
29. Sprenger T, Ruether KV, Boecker $\mathrm{H}$ et al (2007) Altered metabolism in frontal brain circuits in cluster headache. Cephalalgia 27:1033-1042. DOl:https://doi.org/10.1111/j.1468-2982.2007.01386.x

30. Broyd SJ, Demanuele C, Debener S et al (2009) Default-mode brain dysfunction in mental disorders: a systematic review. Neurosci Biobehav Rev 33:279-296.

DOl:https://doi.org/10.1016/j.neubiorev.2008.09.002

31. Morelli N, Pesaresi I, Cafforio G et al (2009) Functional magnetic resonance imaging in episodic cluster headache. J Headache Pain 10:11-14. DOI:https://doi.org/10.1007/s10194-008-0085-z

32. Yang FC, Chou KH, Fuh JL et al (2013) Altered gray matter volume in the frontal pain modulation network in patients with cluster headache. Pain 154:801-807.

DOI:https://doi.org/10.1016/j.pain.2013.02.005

33. Naegel S, Holle D, Desmarattes $\mathrm{N}$ et al (2014) Cortical plasticity in episodic and chronic cluster headache. Neuroimage Clin 6:415-423. DOl:https://doi.org/10.1016/j.nicl.2014.10.003

34. Likova LT, Mineff KN, Nicholas SC (2021) Mental Visualization in the Cerebellum: Rapid Non-motor Learning at Sub-Lobular and Causal Network Levels. Front Syst Neurosci 15:655514. DOl:https://doi.org/10.3389/fnsys.2021.655514

35. Farago P, Szabo N, Toth E et al (2017) Ipsilateral Alteration of Resting State Activity Suggests That Cortical Dysfunction Contributes to the Pathogenesis of Cluster Headache. Brain Topogr 30:281289. DOl:https://doi.org/10.1007/s10548-016-0535-x

36. Moulton EA, Schmahmann JD, Becerra L et al (2010) The cerebellum and pain: passive integrator or active participator? Brain Res Rev 65:14-27. DOl:https://doi.org/10.1016/j.brainresrev.2010.05.005

37. Zhang SS, Wu W, Liu ZP et al (2014) Altered regional homogeneity in experimentally induced low back pain: a resting-state fMRI study. J Neuroeng Rehabil 11:115. DOI:https://doi.org/10.1186/17430003-11-115

38. Kim W, Kim SK, Nabekura J (2017) Functional and structural plasticity in the primary somatosensory cortex associated with chronic pain. J Neurochem 141:499-506.

DOl:https://doi.org/10.1111/jnc.14012

39. Absinta M, Rocca MA, Colombo B et al (2012) Selective decreased grey matter volume of the painmatrix network in cluster headache. Cephalalgia 32:109-115.

DOl:https://doi.org/10.1177/0333102411431334

40. Kiraly A, Szabo N, Pardutz A et al (2018) Macro- and microstructural alterations of the subcortical structures in episodic cluster headache. Cephalalgia 38:662-673.

DOI:https://doi.org/10.1177/0333102417703762

41. Bingel U, Glascher J, Weiller C et al (2004) Somatotopic representation of nociceptive information in the putamen: an event-related fMRI study. Cereb Cortex 14:1340-1345.

DOl:https://doi.org/10.1093/cercor/bhh094

42. Szabo N, Kincses ZT, Pardutz A et al (2013) White matter disintegration in cluster headache. J Headache Pain 14:64. DOI:https://doi.org/10.1186/1129-2377-14-64 
43. Giorgio A, Lupi C, Zhang $\mathrm{J}$ et al (2020) Changes in grey matter volume and functional connectivity in cluster headache versus migraine. Brain Imaging Behav 14:496-504.

DOI:https://doi.org/10.1007/s11682-019-00046-2

44. Brewer JA, Worhunsky PD, Gray JR et al (2011) Meditation experience is associated with differences in default mode network activity and connectivity. Proc Natl Acad Sci USA 108:20254-20259. DOl:https://doi.org/10.1073/pnas.1112029108

45. Hallett M, Di lorio R, Rossini PM et al (2017) Contribution of transcranial magnetic stimulation to assessment of brain connectivity and networks. Clin Neurophysiol 128:2125-2139. DOI:https://doi.org/10.1016/j.clinph.2017.08.007

46. Hodaj H, Alibeu JP, Payen JF et al (2015) Treatment of Chronic Facial Pain Including Cluster Headache by Repetitive Transcranial Magnetic Stimulation of the Motor Cortex With Maintenance Sessions: A Naturalistic Study. Brain Stimul 8:801-807.

DOl:https://doi.org/10.1016/j.brs.2015.01.416

\section{Figures}

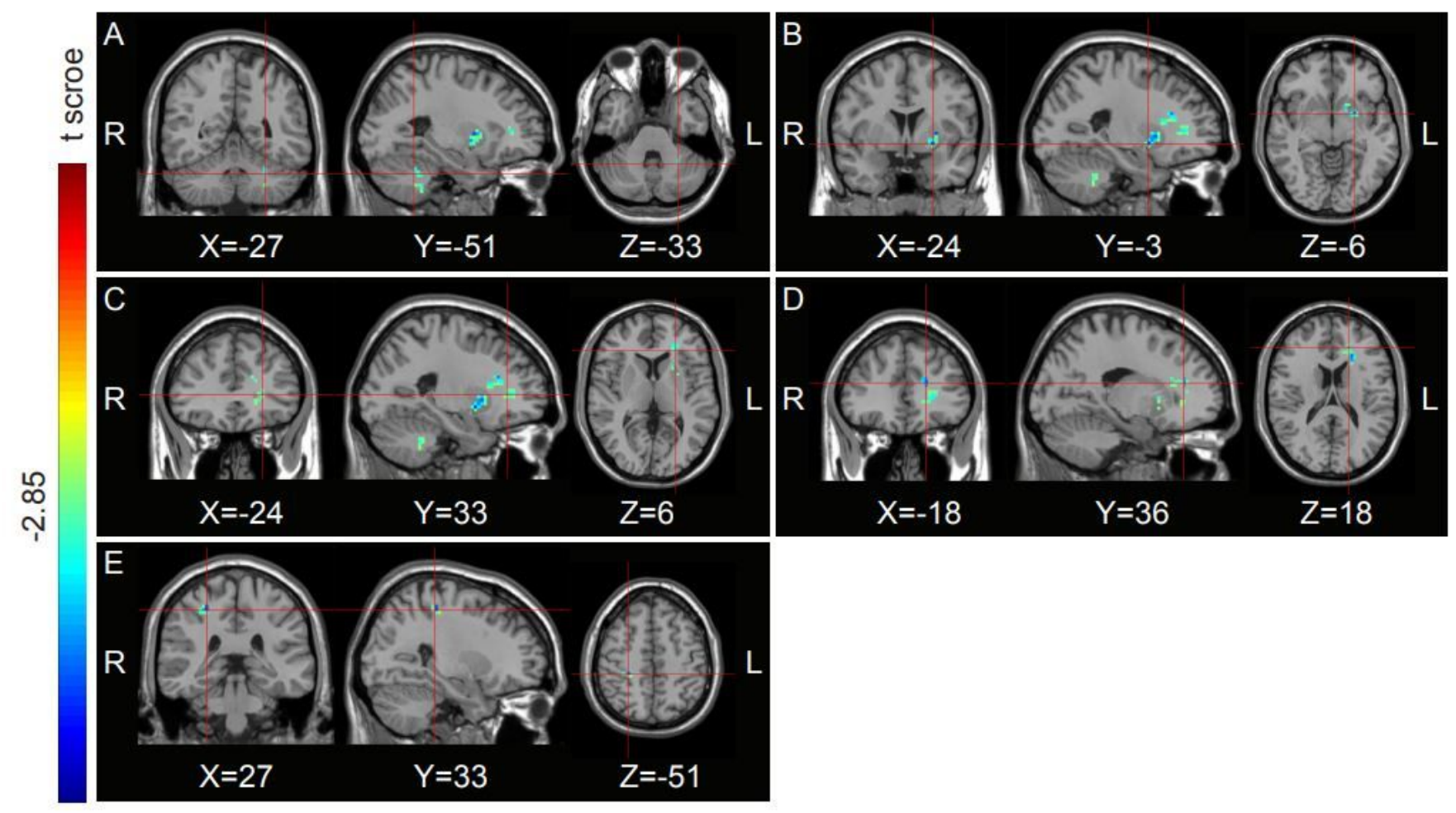

Figure 1 
Regions showing significant differences in fALFF between L-CH patients during the non-painful period and normal controls, with a threshold of $P<0.01$. The color bar indicates the $t$-score of brain regions with fALFF differences between $\mathrm{CH}$ patients during the non-painful period and normal controls, R: right; $\mathrm{L}$ : left.

(A) The left cerebellum $(x=-27, y=-51, z=-33)$. (B) The left lentiform nucleus $(x=-24, y=3, z=-6)$.

(C) The left frontal lobe $(x=-24, y=33, z=6)$. (D) The left anterior cingulate $(x=-18, y=36, z=18)$. (E) The right postcentral gyrus $(x=27 y=-33 z=51)$.

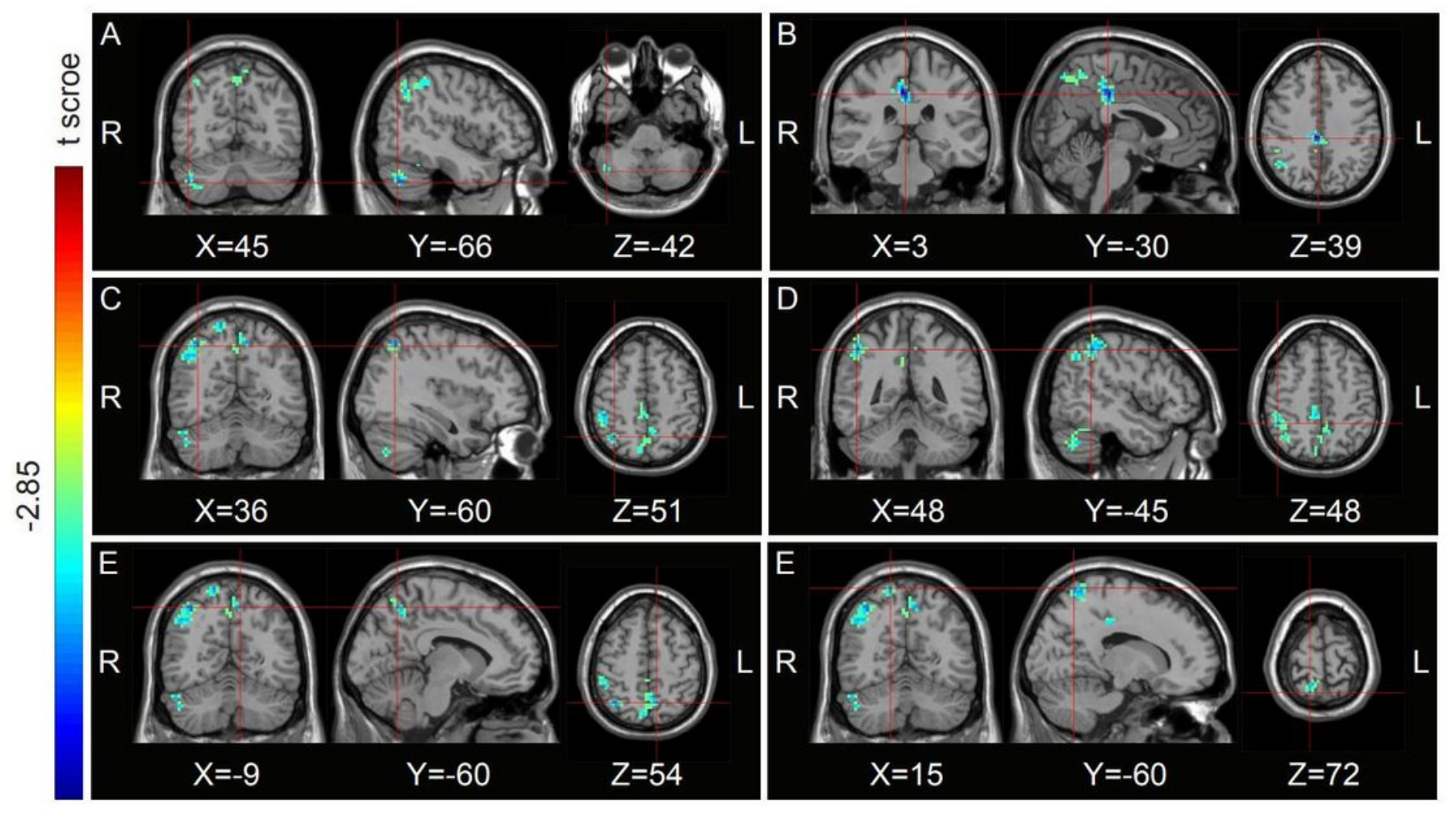

\section{Figure 2}

Regions showing significant differences in fALFF between R-CH patients during the non-painful period and normal controls, with a threshold of $\mathrm{P}<0.01$. The color bar indicates the $t$-score of brain regions with fALFF differences between $\mathrm{CH}$ patients during the non-painful period and normal controls, R: right; $\mathrm{L}$ : left. (A) The right cerebellum $(x=45, y=-66, z=-42)$. (B) The right cingulate gyrus $(x=3, y=-30, z=39)$. (C) The right superior parietal lobule $(x=36, y=-60 z=51)$. (D) The right inferior parietal lobule $(x=48, y=$ $-45, z=48)$. (E) The right postcentral gyrus $(x=-9, y=-60, z=54)$. (F) The left precuneus $(x=15, y=$ $-60, z=72)$. 
A

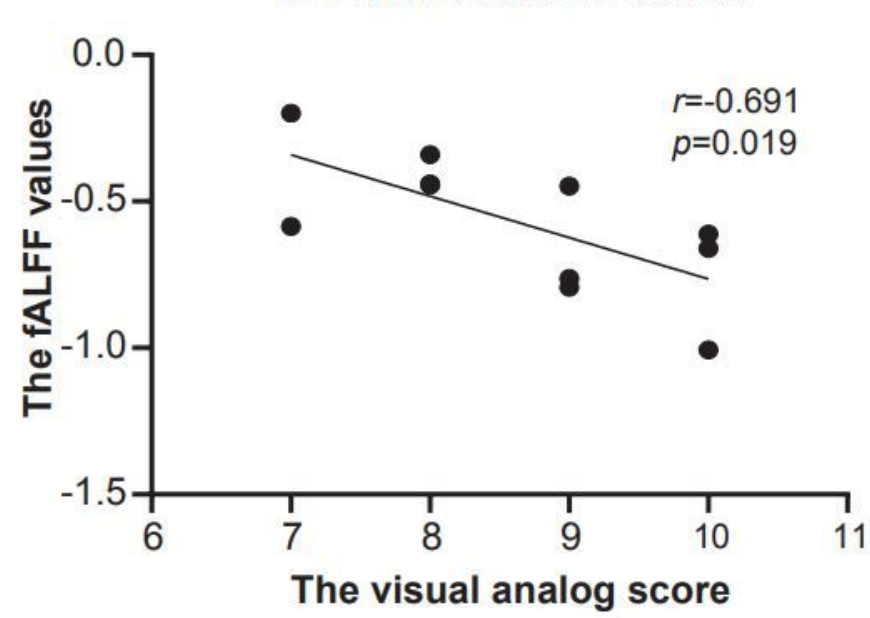

C

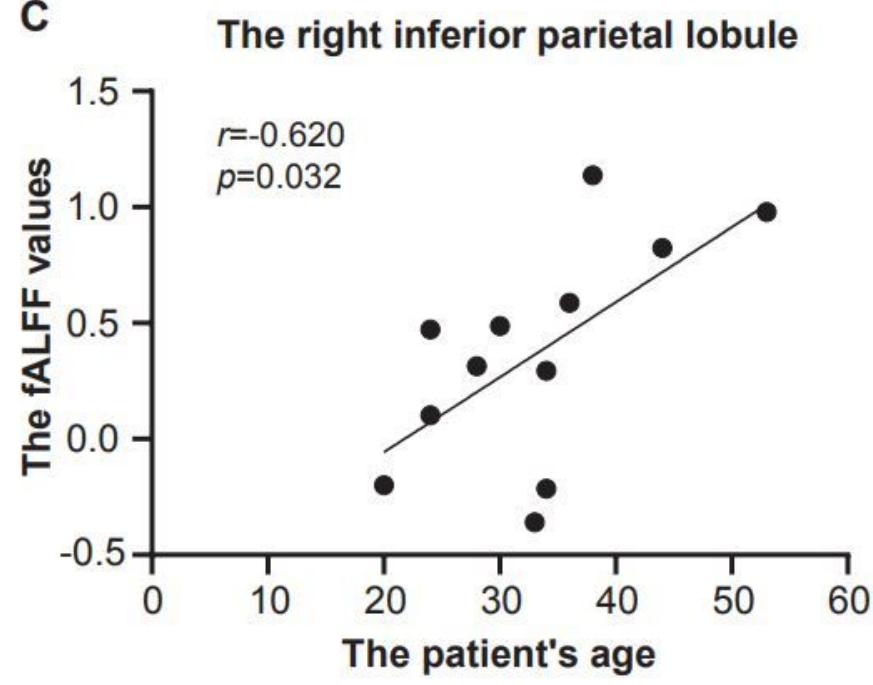

B The right superior parietal lobule

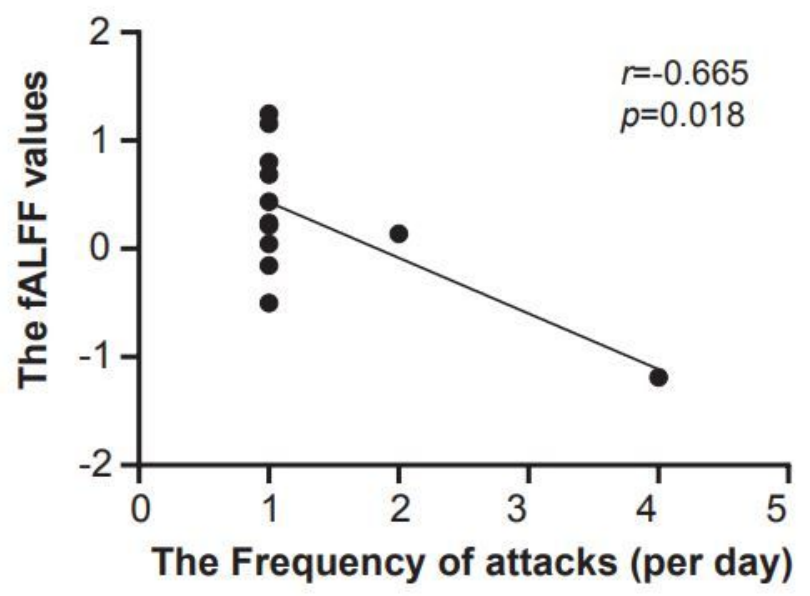

D The right postcentral gyrus

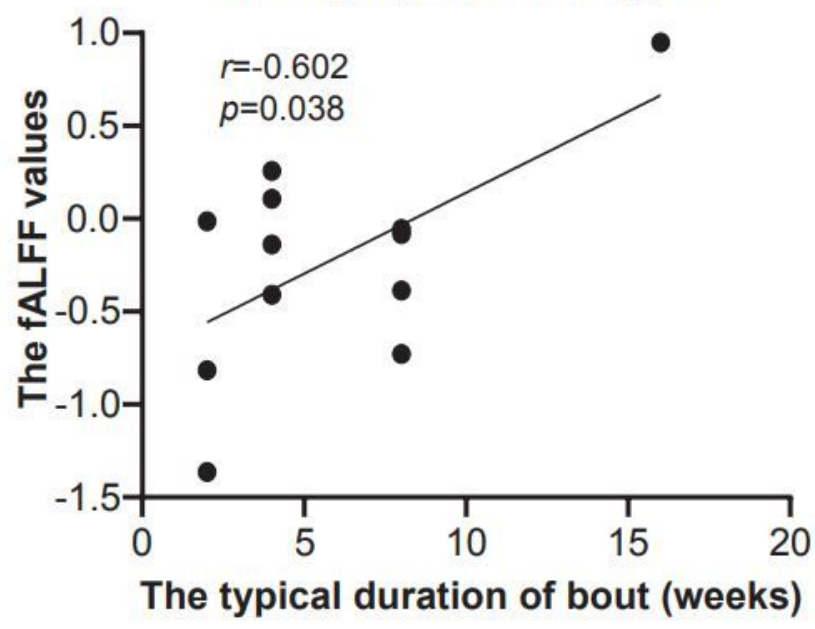

Figure 3

Correlation analysis of clinical characteristics and mean fALFF values. The fALFF value was negatively correlated with the visual analog scale score (A) in the L-CH group and number of attacks per day (B) in the R-CH group, but positively correlated with patient age (C) and the typical duration of bout (in weeks) (D) in the $\mathrm{R}-\mathrm{CH}$ group. 